\title{
Resultado del tratamiento del tumor de Wilms en población pediátrica
}

\author{
NICOLÁS ISA O. ${ }^{1}$, MAURICIO REYES C. ${ }^{1}$, MOISÉS RUSSO N. ${ }^{2,3}$ \\ 1. Oncólogo Radioterapeuta, Instituto Nacional del Cáncer, Servicio de Radioterapia Pediátrica, Santiago de Chile. \\ 2. Residente Radioterapia Oncológica, Instituto de Radiomedicina IRAM, Santiago de Chile. \\ 3. Profesor Asistente, Facultad de Medicina, Universidad Diego Portales.
}

\begin{abstract}
Treatment results of Wilms'tumor in pediatric population

Introduction: Wilms tumor (WT) is the most common form of malignant kidney tumor in childhood. According to PINDA protocols, its treatment includes, depending on stage and presentation, early surgery, radiotherapy (RT) and chemotherapy (CT). The objective of this work is to review the results of all patients of the National Cancer Institute (NCI) with this condition. Patients and Method: A retrospective review of all patients diagnosed with WT at the NCI was conducted. Patient population, RT treatment received and overall survival results were described and prognostic factors were searched. Results: From September 1993 to December 2010, 110 children were treated with RT. The median age at diagnosis was 3.6 years old. Median follow-up was 128 months after RT. In June 2012, out of a total of 107 patients with follow up, 22 had died, 19 due to disease progression. All deaths occurred within three years of treatment. Overall survival at 2, 4 and 12 years old was $82 \%, 78 \%, 78 \%$ respectively. A multivariate analysis showed that each day after surgery and without starting RT, the chances of survival decreased $(\mathrm{p}=.04)$. Conclusions: WT treatment has an excellent prognosis. Survival after 3 years stabilizes without presenting complications, regardless of the group to which the patient belongs. Among the prognostic factors for patients with RT prescription, this radiation should be started early, as close to the surgery as possible.
\end{abstract}

(Key words: Wilms tumor, radiotherapy, cancer, kidney).

Rev Chil Pediatr 2013; 84 (6): 628-633

\section{RESUMEN}

Introducción: El tumor de Wilms (TW) es el tumor renal maligno más frecuente en la infancia, su tratamiento incluye, según su estadio y forma de presentación, cirugía precoz, radioterapia (RT) y quimioterapia (QT) según el protocolo PINDA. El objetivo es conocer los resultados de la serie histórica del Instituto Nacional del

Recibido el 15 de enero de 2013, devuelto para corregir el 12 de marzo de 2013, segunda versión 5 de abril de 2013, tercera versión 28 de abril de 2013, aceptado para publicación el 22 de septiembre de 2013.

Este trabajo cumple con los requisitos sobre consentimiento /asentimiento informado, comité de ética, financiamiento, estudios animales y sobre la ausencia de conflictos de intereses según corresponda.

Correspondencia a:

Nicolás Isa O.

E-mail: dr_isa@hotmail.com 
Cáncer (INC). Pacientes y Método: Revisión retrospectiva de todos los pacientes tratados con el diagnóstico de TW en el INC. Se describe la población de pacientes, tratamiento de RT recibidos, resultados de sobrevida global y factores pronósticos. Resultados: Desde septiembre de 1993 hasta diciembre de 2010 se trataron con RT 110 niños. La mediana de edad al diagnóstico fue de 3,6 años. La mediana de seguimiento fue de 128 meses desde el fin de RT. A junio de 2012, del total de 107 pacientes con seguimiento, 22 habían fallecido, 19 por progresión de enfermedad. Todos los pacientes que fallecieron lo hicieron antes de tres años desde el tratamiento. La sobrevida global a 2, 4, 12 años fue de $82 \%, 78 \%, 78 \%$ respectivamente. En análisis multivariado, cada día después de la cirugía sin comenzar la RT disminuye las probabilidades de sobrevida $(p=0,04)$. Conclusiones: El tratamiento del TW tiene un excelente pronóstico. La sobrevida, luego de los 3 años se estabiliza sin presentar eventos, indistintamente del grupo al que pertenezca el paciente. Dentro de los factores pronósticos, en pacientes con indicación de RT, esta se debe iniciar precozmente, al parecer lo más cercano a la cirugía.

(Palabras clave: Tumor Wilms, radioterapia, cáncer, riñón).

Rev Chil Pediatr 2013; 84 (6): 628-633

\section{Introducción}

El tumor de Wilms (TW) es el tumor renal maligno más frecuente en la infancia, constituyendo alrededor del 5-6\% de los cánceres infantiles ${ }^{1}$. Las indicaciones de su tratamiento incluyen, según su estadio y forma de presentación, cirugía precoz, radioterapia (RT) y quimioterapia (QT). En Chile, el tratamiento se realiza según las indicaciones del protocolo PINDA de $\mathrm{TW}^{1}$, que tiene como referencia el protocolo del National Wilms Tumor Study Group de Estados Unidos (NWTS 3-5) ${ }^{2-9}$. Las indicaciones de tratamiento incluyen la cirugía primaria de entrada y quimioterapia adyuvante en el $100 \%$ de los pacientes. Las indicaciones de RT consisten en que el día de la nefrectomía será considerado como el día 0 , todos los pacientes con una masa renal serán inscritos para RT al diagnóstico, la que será suspendida si no corresponde según la etapa y la histología. Los pacientes recibirán una dosis total de 10,8 Gy en 6 fracciones de 1,8 Gy (dosis diaria de $1,8 \mathrm{~Gy} /$ fracción por 5 días a la semana). La fracción diaria al tumor deberá disminuirse a 1,5 Gy cuando se incluyen grandes volúmenes en los campos (abdomen total) o cuando se irradia pulmón, que se dará en 7 fracciones (1,5 Gy/fracción por 5 veces a la semana). La RT debe iniciarse no después del $9^{\circ}$ día. Para histología favorable, Etapas I y II no deberán irradiarse, Etapa III todos se irradiarán en el postoperatorio, finalmente para Etapa IV se distribuye RT infra diafragmática sólo si el tumor primario se catalogó como etapa III; y las dosis y campos son los mismos, sólo las metástasis abdominales que no son resecables por su localización o su extensión recibirán RT 19,8 Gy en 11 fracciones (1,8 Gy/día). Los pacientes con metástasis pulmonares al diagnóstico, deben tratarse sin considerar el número y la localización de las metástasis visibles a la Rx de tórax. El campo de tratamiento incluye ambos pulmones, en especial los ápices y las porciones inferiores y posteriores y se distribuyen 12 Gy en 8 fracciones. Las metástasis pulmonares localizadas que persistan 2 semanas después de haber distribuido 12 Gy pueden ser resecadas quirúrgicamente o se pueden irradiar con una dosis adicional de 7,5 Gy (1,5 Gy/día por 5 días). Para tumor de histología anaplásica recibirán RT desde Etapa II en adelante y para histología de células claras o rabdoide, recibirán RT desde Etapa I, igual que la descrita anteriormente. Sólo se reserva quimioterapia neoadyuvante a los pacientes que no pueden recibir cirugía de entrada.

El objetivo de esta publicación es conocer los resultados de la serie histórica del Instituto Nacional del Cáncer (INC), centro de referencia nacional para RT pediátrica desde el año 1993.

\section{Pacientes y Método}

Revisión retrospectiva de todos los pacientes tratados con RT con el diagnóstico de TW 
en el INC, rescatando ficha, informe de derivación, exámenes adjuntos, informe de radioterapia e informe de alta de tratamiento de los pacientes. El análisis consideró los pacientes entre el período desde septiembre de 1993, primer paciente registrado, a diciembre de 2010 y así obtener un seguimiento mínimo de al menos 2 años.

Se tabularon todos los datos posibles de obtener, entre ellos fecha de nacimiento, fecha de diagnóstico, estadio al diagnóstico, ubicación, histología, riesgo, tratamiento primario efectuado, fecha y características de la cirugía, fecha y características de la quimioterapia, fecha y características de la radioterapia. Durante el seguimiento se exploraron las recidivas y sus características y tratamiento (s) de rescate y estado al último control. El análisis de sobrevida se realizó mediante el método de KaplanMeier. Comparaciones de sobrevida entre grupos se realizaron mediante el método de log-rank. Comparaciones de características entre grupos se realizaron mediante t-student o Rank-sum según fuese apropiado. Exploraciones de factores pronósticos relevantes se realizaron con análisis univariado y multivariado con regresión de Cox. Todos los análisis fueron realizados en STATA 12.

\section{Resultados}

Desde septiembre de 1993 hasta diciembre de 2010 se trató con RT 110 niños con el diagnóstico de TW. La mediana de edad al diagnóstico fue de 3,6 años (rango de 0,4-13,3 años); Las características de los pacientes se resumen en la tabla 1.

Dentro de las características del tratamiento inicial, este consistió en cirugía en 60 pacientes y QT en 50 pacientes. Los que recibieron cirugía inicial tendieron estadísticamente a ser de

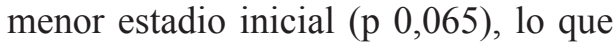
se correlaciona con los que no pudieron recibir cirugía de entrada como lo indi- ca el protocolo PINDA, por imposibilidad de cirugía dado el tamaño y compromiso tumoral por lo que se prefiere quimioterapia neoadyuvante. Tanto la cirugía, QT y RT (tiempo, campos, dosis, etc.), se realizaron según las exigencias del protocolo PINDA ${ }^{1}$.

En cuanto a las características de la dosimetría de la RT, esta fue 2D en un principio y luego con la implementación de TAC, pasó a ser RT conformacional 3D, en donde las dosis y tamaños y características de los campos no variaron. Los campos de tratamiento consistieron en Abdomen total en 31 pacientes $(28,1 \%)$, Hemiabdomen derecho 31 pacientes $(28,1 \%)$, Hemiabdomen izquierdo 23 pacientes (21\%), Toraco Abdominal 18 pacientes (16,4\%), Pulmonar total $7(6,4 \%)$; de ellos 79 pacientes no recibieron boost ( $72 \%$ ) y 31 sí (28\%). Las características de la RT se resumen en la tabla 2.

El 100\% de los pacientes recibieron Quimioterapia según lo indica el protocolo PINDA ${ }^{1}$.

Se perdieron 3 pacientes luego del alta de RT, para efectos estadísticos, estos pacientes fueron censurados en la fecha de su último control.

La mediana de seguimiento fue de 128 meses (mínimo de 18 meses) desde el fin de tratamiento de la RT.

Durante el seguimiento, 19 pacientes presentaron recidivas locales y a distancia, estas últimas principalmente en los pulmones, 12 de ellos recibieron RT como parte de su tratamiento de rescate. De ellos, 6 pacientes se encuentran sin evidencia de enfermedad actual y los otros 6 fallecidos. Sus características iniciales y tratamiento de rescate se resumen en la tabla 3 .

Tabla 1. Características de los pacientes

\begin{tabular}{|llrr|}
\hline \multirow{3}{*}{ Sexo } & & n & \% \\
& Hombres & 41 & 37 \\
Riñón & Mujeres & 69 & 63 \\
& Derecho & 54 & 49 \\
& Izquierdo & 51 & 46 \\
& Ambos & 5 & 5 \\
Estadio y & I favorable (recidiva) & 1 & 1 \\
riesgo & desfavorable (sarcoma de células claras) & 2 & 2 \\
& II favorable (recidiva) & 2 & 2 \\
& desfavorable & 5 & 5 \\
& III favorable & 50 & 45 \\
& desfavorable & 12 & 11 \\
& IV favorable & 30 & 27 \\
& desfavorable & 3 & 3 \\
& V favorable & 5 & 4 \\
Total & & 110 & 100 \\
\hline
\end{tabular}


Tabla 2. Características de la Radioterapia

\begin{tabular}{|c|c|c|c|c|c|c|c|}
\hline $\begin{array}{l}\text { Campos de } \\
\text { tratamiento }\end{array}$ & $\begin{array}{c}\mathbf{n} \text { de } \\
\text { pacientes }\end{array}$ & $\begin{array}{l}\text { Dosis de } \\
\text { tratamiento }\end{array}$ & $\begin{array}{l}\text { Fracciona- } \\
\text { miento }\end{array}$ & Boost & $\begin{array}{c}n \text { de } \\
\text { pacientes } \\
\text { con boost }\end{array}$ & Fotones & $\begin{array}{c}\% \text { total de } \\
\text { pacientes }\end{array}$ \\
\hline Abdomen total & 31 & $10,8(5,4-10,8)$ & $1,8(1,5-1,8)$ & $5,4(3-14,4)$ & 8 & $6(1,25-6)$ & 28,1 \\
\hline $\begin{array}{l}\text { Hemiabdomen } \\
\text { derecho }\end{array}$ & 31 & $10,8(10,5-10,8)$ & $1,8(1,5-1,8)$ & $5,4(3,6-5,4)$ & 3 & $6(1,25-18)$ & 28,1 \\
\hline $\begin{array}{l}\text { Hemiabdomen } \\
\text { izquierdo }\end{array}$ & 23 & $10,8(10,5-10,8)$ & $1,8(1,5-1,8)$ & 3,6 & 1 & $6(1,25-6)$ & 21 \\
\hline Toraco abdominal & 18 & $10,5(10,5-12)$ & $1,5(1,5-1,8)$ & $1,5(1,5-10,8)$ & 16 & & 16,4 \\
\hline Pulmonar total & 7 & 12 & 1,5 & $9 \quad(5,4-10,8)$ & 3 & $6(1,25-6)$ & 6,4 \\
\hline Total & 110 & & & & 31 & & $100 \%$ \\
\hline
\end{tabular}

Tabla 3. Tratamiento de pacientes que presentaron recidiva

\begin{tabular}{|c|c|c|c|c|c|}
\hline $\begin{array}{l}\text { Estadio } \\
\text { inicial }\end{array}$ & $\begin{array}{l}\text { Campo de } \\
\text { tratamiento } \\
\text { inicial }\end{array}$ & $\begin{array}{c}\text { Dosis de } \\
\text { tratamiento y boost }\end{array}$ & $\begin{array}{l}\text { Campo de } \\
\text { recidiva }\end{array}$ & $\begin{array}{l}\text { Dosis de } \\
\text { recidiva y boost }\end{array}$ & $\begin{array}{c}\text { Estado al } \\
\text { último } \\
\text { control }\end{array}$ \\
\hline I (recidiva) & $\begin{array}{l}\text { Hemiabdomen } \\
\text { derecho }\end{array}$ & 10,8 a $1,8+5,4$ a 1,8 & Pulmones & 12 a 1,5 & SEE \\
\hline III (favorable) & $\begin{array}{l}\text { Abdomen } \\
\text { total }\end{array}$ & 10,8 a 1,8 & Pulmones & 12 a $1,5+10,8$ a 1,8 & Fallecido \\
\hline III (favorable) & $\begin{array}{l}\text { Hemiabdomen } \\
\text { izquierdo }\end{array}$ & 10,8 a 1,8 & Pulmones & 12 a 1,5 & SEE \\
\hline III (favorable) & $\begin{array}{l}\text { Hemiabdomen } \\
\text { derecho }\end{array}$ & 10,8 a 1,8 & Pulmones & 12 a 1,5 & Fallecido \\
\hline III (favorable) & $\begin{array}{l}\text { Hemiabdomen } \\
\text { derecho }\end{array}$ & 10,8 a 1,8 & Toraco abdominal & 10,5 a $1,5+1,5$ a 1,5 & SEE \\
\hline III (favorable) & $\begin{array}{l}\text { Hemiabdomen } \\
\text { izquierdo }\end{array}$ & 10,8 a 1,8 & Pulmones & 12 a $1,5+7,5$ a 1,5 & Fallecido \\
\hline III (favorable) & $\begin{array}{l}\text { Hemiabdomen } \\
\text { derecho }\end{array}$ & 10,8 a 1,8 & Pulmones & 12 a 1,5 & Fallecido \\
\hline III (favorable) & $\begin{array}{l}\text { Hemiabdomen } \\
\text { derecho }\end{array}$ & 10,8 a $1,8+3,6$ a 1,8 & $\begin{array}{l}\text { Hemiabdomen } \\
\text { derecho }\end{array}$ & 10,8 a $1,8+14,4$ a 1,8 & SEE \\
\hline IV (favorable) & $\begin{array}{l}\text { Abdomen } \\
\text { total }\end{array}$ & 5,4 a $1,8+5,4$ a 1,8 & Pulmones & 12 a 1,5 & SEE \\
\hline IV (favorable) & $\begin{array}{l}\text { Hemiabdomen } \\
\text { izquierdo }\end{array}$ & 10,8 a 1,8 & Pulmones & 12 a 1,5 & SEE \\
\hline IV (favorable) & $\begin{array}{l}\text { Hemiabdomen } \\
\text { derecho }\end{array}$ & 10,8 a 1,8 & Pulmones & 12 a 1,5 & Fallecido \\
\hline V (favorable) & $\begin{array}{l}\text { Abdomen } \\
\text { total }\end{array}$ & 10,8 a $1,8+14,4$ a 1,8 & $\begin{array}{l}\text { Pulmonar e } \\
\text { hipogástrica }\end{array}$ & 12 a 1,5 y 10,8 a 1,8 & Fallecido \\
\hline
\end{tabular}

A junio de 2012, del total de 107 pacientes con seguimiento, 22 pacientes se encontraban fallecidos. Las causas de muerte fueron progresión de enfermedad en 19 pacientes, leucemización en un paciente (complicación atribuida a la QT), fibrosis pulmonar en un paciente (complicación atribuida a la RT toraco abdominal) y un último paciente por Trombosis Mesentérica.

En el análisis, la sobrevida global a 2, 4, 12 años fue de alrededor del 82\%, 78\%, 78\% respectivamente. La curva de sobrevida global se adjunta en la figura 1 y separada por estadio en la figura 2. 


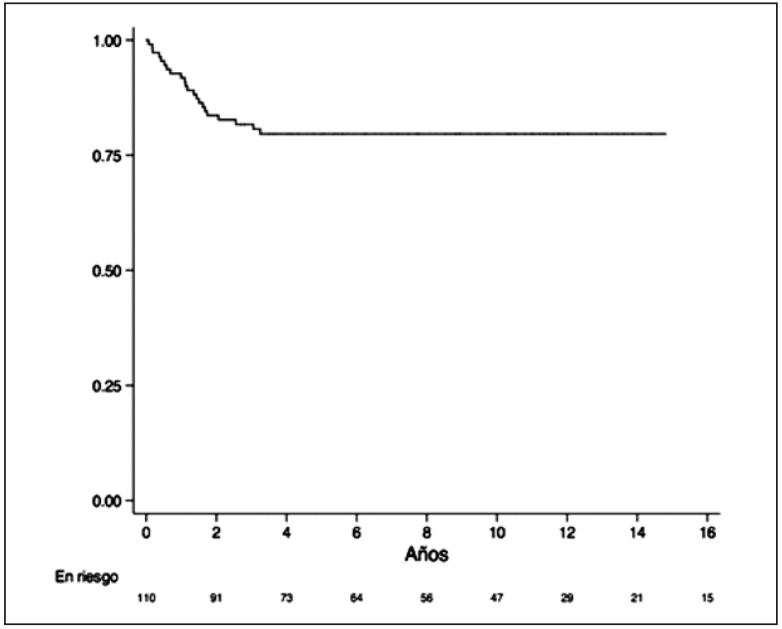

Figura 1. Sobrevida global.

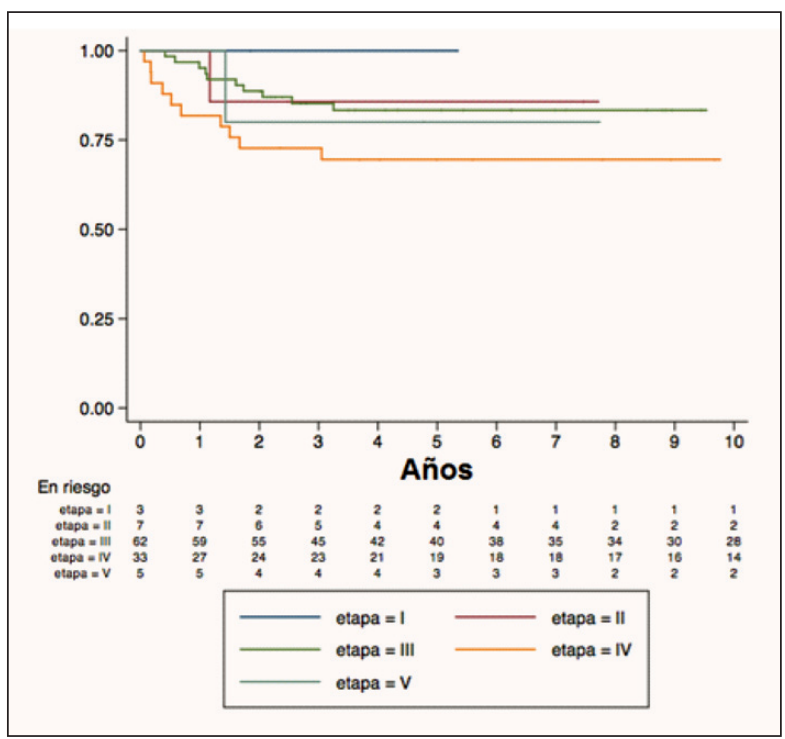

Figura 2. Sobrevida global por grupos.

Tabla 4. Análisis multivariado

\begin{tabular}{|lcc|}
\hline & HR & p \\
\hline Edad >3,5 años (mediana de edad) & 1,35 & 0,494 \\
\hline Estadio IV vs III & 2,1 & 0,09 \\
\hline $\begin{array}{l}\text { Días desde la cirugía a inicio de radioterapia } \\
\text { (9 días o más) }\end{array}$ & 1,02 & 0,96 \\
$\begin{array}{l}\text { Días desde cirugía a inicio radioterapia } \\
\text { (variable continua) }\end{array}$ & 1,01 & 0,04 \\
\hline
\end{tabular}

Dentro del análisis estadístico se intentaron buscar posibles factores pronósticos de sobrevida. Se exploraron la edad, estadio III vs IV, días desde la cirugía a RT como variable dicotómica de hasta 9 días o más, tal como lo exige el protocolo PINDA, en donde no deben transcurrir más de 9 días desde la cirugía para comenzar la Radioterapia, y así evaluar el impacto diario del retraso de RT en el pronóstico. Al análisis univariado y multivariado, sólo este último criterio resultó ser positivo, lo que nos muestra que cada día después de la cirugía sin comenzar la RT es peor $(p=0,04)$. Los resultados del análisis multivariado se resumen en la tabla 4.

\section{Discusión}

El TW es un tumor que afortunadamente con tratamiento multi asociado, tiene un excelente pronóstico. Esto se evidenció en nuestra serie, la más grande y con mayor seguimiento de TW, publicada por una sola institución.

Dentro del análisis de sobrevida de los gráficos 1 y 2 , se aprecia que la mayoría de los eventos adversos ocurren durante los primeros 3 años desde el fin de la RT y luego la curva de sobrevida se estabiliza sin presentar eventos, manteniéndose en alrededor del $80 \%$ para todos los estadios. De estos datos de desprende que los pacientes que van a fallar lo harán en forma precoz y que su tratamiento de rescate será determinante en el pronóstico de sobrevi$\mathrm{da}$, indistintamente del grupo al que pertenezca el paciente.

El tratamiento del TW es seguro, ya que sólo 2 de los 110 pacientes fallecieron a causa de este. Así mismo, evidenciamos que la RT es un tratamiento seguro y efectivo, incluso cuando incluye a los pulmones tal como lo muestran las publicaciones internacionales ${ }^{7-9}$.

Dentro de los factores pronósticos, evidenciamos que al parecer hay que realizar la RT lo más cercano a la Cirugía para no comprometer el resultado de esta.

E1 TW es poco frecuente, pero con ade- 
cuado tratamiento es una enfermedad de muy buen pronóstico. El hecho de tener protocolizado su tratamiento a nivel nacional garantiza un tratamiento de calidad, comparable con las mejores series internacionales ${ }^{7-9}$.

\section{Referencias}

1.- Protocolo Tumor de Wilms, Protocolo Pinda 99, Versión 2005, Chile.

2.- D'Angio G, Breslow J, Beckwith J: Treatment of Wilms' tumor: Results of the Third National Wilms' Tumor Study. Cancer 1989; 64: 349-60.

3.- Green D, Breslow N, Beckwith J, et al: Comparison between single-dose and divided-dose administration of dactinomycin and doxorubicin for patients with Wilms' tumour: a report from the national Wilms' tumour study group. J Clin Oncol 1998; 16: 237-45.

4.- Green D, Breslow N, Beckwith J, et al: Effect of duration of treatment on treatment outcome and cost of treatment for Wilms' tumour: a report from the national
Wilms' tumour study group. J Clin Oncol 1998; 16 : 3744-51.

5.- Shamberger R, Grundy P, Green D: Nephrectomy only for very low risk Wilms' tumour (VLRWT). Pediatr Blood Cancer 2005; 45 (4): 379 (abstract).

6.- Green D, Breslow N, Evans I, Moksness J, D’Angio G: Treatment of children with stage IV favourable histology Wilms' tumour: a report from the national Wilms' tumour study group. Med Pediatr Oncol 1996; 26: 147-52.

7.- Meisel J, Guthrie K, Breslow N, et al: Significance and management of computed tomography detected pulmonary nodules: a report from the national Wilms' tumour study group. Int J Radiat Oncol Biol 1999; 44: 579-85.

8.- Ahmed H, Arya M, Tsiouris A, et al: An update on the management of Wilms' tumour. EJSO 2007; 33: 824-31.

9.- Nicolin G, Taylor R, Baughan C, et al: Outcome after pulmonary radiotherapy in Wilms' tumor patients with pulmonary metastases at diagnosis: a UK children's cancer study group, Wilms' tumour working group study. Int J Radiation Oncology Biol Phys 2008; 70 (1): 175-80. 